\title{
AN UPWINDING MIXED FINITE ELEMENT METHOD FOR A MEAN FIELD MODEL OF SUPERCONDUCTING VORTICES ${ }^{*, * *}$
}

\author{
Zhiming Chen $^{1}$ AND Qiang Du ${ }^{2}$
}

\begin{abstract}
In this paper, we construct a combined upwinding and mixed finite element method for the numerical solution of a two-dimensional mean field model of superconducting vortices. An advantage of our method is that it works for any unstructured regular triangulation. A simple convergence analysis is given without resorting to the discrete maximum principle. Numerical examples are also presented.
\end{abstract}

Mathematics Subject Classification. 65N99, 82D55.

Received: March 16, 1999. Revised: September 14, 1999.

\section{InTRODUCTION}

Recently, the mathematical analysis and the numerical studies of the mean field models have received much attention [3-5, 8, 11, 13, 14, 17, 19, 20,22]. Vortex simulations based on the mean field models provide possible alternatives to the popular yet limited simulations based on the Ginzburg-Landau models [6,9,10]. In the two dimensional setting, the mean field model that we are interested in can be described, after proper scaling, as follows:

$$
\begin{aligned}
& \omega_{t}-\nabla \cdot(\omega \nabla u)=0 \quad \text { in } \Omega_{T}, \\
& -\Delta u+u=\omega \quad \text { in } \Omega_{T} .
\end{aligned}
$$

Here, $\omega$ and $u$ represent the vortex density and the average magnetic field, respectively. The superconducting sample is assumed to occupy a convex polygonal domain $\Omega \subset \mathbb{R}^{2}$ with boundary $\Gamma$. $\Omega_{T}=\Omega \times(0, T)$.

In $[17,22]$, the existence and uniqueness of solutions and the regularity estimates are obtained for solutions with compact support. In $[8,14]$, some hybrid finite element/finite volume/finite difference approximations have also been proposed and analyzed. So far, the only complete convergence theory available in the literature for the two dimensional mean field model is that presented in [8]. However, to apply the finite volume based integration schemes as in $[8,14]$, the triangular grids need to satisfy the local equiangular assumption in order to preserve the discrete maximum principle.

\footnotetext{
Keywords and phrases. Mean field model, superconductivity, vortices, mixed finite element, unstructured grid, convergence analysis.

* The work of the first author was partially supported by the National Natural Science Foundation of China under the grant No.19771080 and China National Key Project "Large Scale Scientific and Engineering Computing".

** The research of the second author was supported in part by NSF under Grant DMS-9796208 and a grant from HKRGC.

1 Institute of Mathematics, Academia Sinica, Beijing 100080, P.R. China. e-mail: zmchen@math03.math.ac.cn

2 Department of Mathematics, Hong Kong University of Science and Technology, Clear Water Bay, Hong Kong, and Department of Mathematics, Iowa State University, Ames, IA 50011, USA. e-mail: madu@uxmail.ust.hk
} 
In this paper, we present a method that can be applied to general unstructured triangular grids. To approximate the hyperbolic equation for the vortex density (1.1), our basic idea is to use the upwinding finite element method developed in [18] for solving the neutron transport equation

$$
\boldsymbol{\beta} \cdot \nabla w+\sigma w=f \quad \text { in } \Omega,
$$

where $\boldsymbol{\beta}$ is a constant vector in $\mathbb{R}^{2}$ and $\sigma$ is a given function such that $\sigma \geq \sigma_{0}>0$ for some constant $\sigma_{0}$. For a given triangular grid, the discretization involves the normal component of $\beta$ on the triangular edges. If the standard conforming piecewise linear finite elements on the triangular grids are used to solve $(1.2)$ as in $[8,14]$ and the upwinding scheme in [18] is extended to solve (1.1), then we encounter the difficulty that the normal components of $\nabla u_{h}$ across any inter-element boundaries are in general discontinuous. Due to this observation, we propose in this paper to consider mixed finite element method to approximate (1.2). In particular, the mixed Raviart-Thomas finite element approximation is used to solve the magnetic field equation (1.2). A simple convergence analysis of the numerical solutions to the weak solution of the two dimensional mean field model (1.1-1.2) can be completed for any regular quasi-uniform triangulation of $\Omega$ without use of the discrete maximum principle.

The paper is organized as follows. In Section 2, the weak formulation of the model is provided. A numerical method using upwinding mixed finite element approximations is presented in Section 3. The main theorem is stated in Section 4. A number of technical estimates on the discrete solutions are given in Section 5 . The proof of the main theorem is given in Section 6. Discussion of the full-discrete approximations is made in Section 7. Some preliminary numerical experiments are presented in Section 8. Further remarks and comments are given in Section 9.

\section{WEAK FORMULATION}

Let $(\cdot, \cdot)$ and $(\cdot, \cdot)_{\mathcal{D}}$ denote the standard $\mathrm{L}^{2}$ inner products on $\Omega$ and on any two dimensional domain $\mathcal{D}$ respectively. Let $\mathrm{H}_{0}^{1}(\Omega)$ and $\mathrm{H}^{-1}(\Omega)$ denote the standard Sobolev space and the dual space of functions of the variable $\mathbf{x} \in \Omega$. We also introduce the subspace of $\mathrm{L}^{2}(\Omega)^{2}$ :

$$
\mathrm{H}(\operatorname{div} ; \Omega)=\left\{\mathbf{q} \in \mathrm{L}^{2}(\Omega)^{2}: \operatorname{div} \mathbf{q} \in \mathrm{L}^{2}(\Omega)\right\} .
$$

For any Banach space $B$, its norm is denoted by $\|\cdot\|_{B}$.

For the system (1.1-1.2), we consider the initial condition

$$
\left.\omega\right|_{t=0}=\omega_{0} \geq 0 \quad \text { in } \Omega,
$$

and the boundary condition

$$
u=H_{\text {ext }} \geq 0 \quad \text { on } \partial \Omega_{T},
$$

where $\partial \Omega_{T}=\{(\mathbf{x}, t): \mathbf{x} \in \partial \Omega, t \in(0, T)\}$ and $H_{\text {ext }}$ is an external applied magnetic field. For convenience, let us introduce $\mathbf{p}=-\nabla u$.

For simplicity, we consider only the case where the solution $\omega$ has a compact support throughout the time interval $(0, T)$ of interests. We also assume that $H_{\text {ext }}$ is a constant in time and space. The results of this paper can be easily extended to general non-constant functions $H_{\text {ext }}$. Finally, we assume $\omega_{0}$ is nonnegative and $\omega_{0} \in \mathrm{L}^{\infty}(\Omega)$. Note that for a given $T$ and any given initial vortex density that is $\mathrm{L}^{\infty}$ bounded with a compact support, if the domain $\Omega$ is suitably large, then the vortex density will have compact support in $(0, T)$. 
The weak form is given by: find $(\omega, u, \mathbf{p})$ with $\omega \in \mathrm{H}^{1}\left(0, T ; \mathrm{H}^{-1}(\Omega)\right) \cap \mathrm{L}^{2}\left(\Omega_{T}\right), u \in \mathrm{L}^{2}\left(0, T ; \mathrm{L}^{2}(\Omega)\right)$ and $\mathbf{p} \in \mathrm{L}^{2}(0, T ; \mathrm{H}(\operatorname{div} ; \Omega))$ such that

$$
\begin{aligned}
& \left(\omega_{0}, \phi(\cdot, 0)\right)-\int_{0}^{T}\left(\omega, \phi_{t}+\mathbf{p} \cdot \nabla \phi\right) \mathrm{d} t=0, \quad \forall \phi \in \mathrm{C}_{0}^{\infty}(\Omega \times[0, T)) \\
& \int_{0}^{T}[(\operatorname{div} \mathbf{p}, \varphi)+(u-\omega, \varphi)] \mathrm{d} t=0, \quad \forall \varphi \in \mathrm{L}^{2}\left(0, T ; \mathrm{L}^{2}(\Omega)\right) \\
& \int_{0}^{T}\left[(\mathbf{p}, \mathbf{q})-\left(u-H_{\text {ext }}, \operatorname{div} \mathbf{q}\right)\right] \mathrm{d} t=0, \quad \forall \mathbf{q} \in \mathrm{L}^{2}(0, T ; \mathrm{H}(\operatorname{div} ; \Omega)) .
\end{aligned}
$$

\section{Finite ELEMENT APPROXimations}

Let $\Sigma_{h}$ denote a regular, quasi-uniform triangulation of $\Omega$, with $h$ being the typical mesh parameter [1]. Let $\{K\}$ denote the triangles and $\{\gamma\}$ denote the edges. Let $E_{h}$ be the collection of all inter-element edges (that is, excluding those on the boundary $\Gamma$ ).

Let $P_{h} \subset \mathrm{H}(\operatorname{div} ; \Omega)$ denote the lowest order Raviart-Thomas element space and $Q_{h} \subset \mathrm{L}^{2}(\Omega)$ be the space of piecewise constants with respect to $\Sigma_{h}$. We now approximate $u$ and $\omega$ by elements of $Q_{h}$ and approximate $\mathbf{p}$ by elements of $P_{h}$.

For each edge $\gamma$ of a triangle $K$, we define the outward normal vector by $\mathbf{n}$. It is easily seen that the element $\mathbf{p}_{h}$ of $P_{h}$ is uniquely determined by

$$
\left\{\mathbf{p}_{h}\left(\mathbf{x}_{\gamma}\right) \cdot \mathbf{n}\left(\mathbf{x}_{\gamma}\right) \mid \mathbf{x}_{\gamma} \text { is the midpoint of some edge } \gamma \text { of } \Sigma_{h}, \mathbf{n} \perp \gamma\right\} .
$$

Given $\mathbf{p}_{h}$, we divide the boundary $\partial K$ of each element $K \in \Sigma_{h}$ into two parts

$$
\begin{array}{ll}
\partial K_{-}=\cup\left\{\gamma \subset \partial K: \mathbf{p}_{h}\left(\mathbf{x}_{\gamma}\right) \cdot \mathbf{n}<0\right\} & \text { inflow }, \\
\partial K_{+}=\cup\left\{\gamma \subset \partial K: \mathbf{p}_{h}\left(\mathbf{x}_{\gamma}\right) \cdot \mathbf{n} \geq 0\right\} & \text { outflow } .
\end{array}
$$

Next, for a fixed $\mathbf{p}_{h}$, we define the upwind value of any $\eta_{h} \in Q_{h}$ on $\partial K$ as

$$
\tilde{\eta}_{h}= \begin{cases}\eta_{h}^{+}\left(\text {interior trace of } \eta_{h}\right) & \text { on } \partial K_{+} \\ \eta_{h}^{-}\left(\text {exterior trace of } \eta_{h}\right) & \text { on } \partial K_{-}\end{cases}
$$

and assume $\eta_{h}^{-}=0$ on $\partial K_{-} \cap \Gamma$. For any $\gamma \in E_{h}$, we define the jump of $\eta_{h} \in Q_{h}$ across $\gamma$ by

$$
\left[\eta_{h}\right]:=\eta_{h}^{+}-\eta_{h}^{-} .
$$

The numerical method we consider in this paper is based on an upwinding finite element scheme for (2.3) and the mixed finite element approximation using the Raviart-Thomas element for (1.1), (1.2) and $\mathbf{p}=-\nabla u$ as follows.

For $\left\{\omega_{h}, u_{h}, \mathbf{p}_{h}\right\}$ in $Q_{h} \times Q_{h} \times P_{h}$, for each $t \in(0, T]$, we have for each $t \in(0, T]$ that

$$
\begin{gathered}
\left(\frac{\mathrm{d} \omega_{h}}{\mathrm{~d} t}, \eta_{h}\right)_{K}+\int_{\partial K}\left(\mathbf{p}_{h} \cdot \mathbf{n}\right) \tilde{\omega}_{h} \eta_{h} \mathrm{~d} \gamma=0 \quad \forall \eta_{h} \in Q_{h}, \quad \forall K \in \Sigma_{h}, \\
\left(\operatorname{div} \mathbf{p}_{h}, v_{h}\right)+\left(u_{h}-\omega_{h}, v_{h}\right)=0 \quad \forall v_{h} \in Q_{h} \\
\left(\mathbf{p}_{h}, \mathbf{q}_{h}\right)-\left(u_{h}-H_{\text {ext }}, \operatorname{div} \mathbf{q}_{h}\right)=0 \quad \forall \mathbf{q}_{h} \in P_{h} .
\end{gathered}
$$


The initial condition is given by:

$$
\left(\omega_{h, 0}, v_{h}\right)=\left(\omega_{0}, v_{h}\right), \quad \forall v_{h} \in Q_{h} .
$$

The existence of a unique solution to the discrete problem (3.1)-(3.4) follows from the standard ODE theory by using the uniform in time a priori estimate in Corollary 5.6 to be proved later. Here we omit the details.

\section{The CONVERGENCE THEOREM}

For brevity, we first focus on the semi-discrete approximation. Fully discrete approximations are considered later. We now state the main theorem:

Theorem 4.1. (Convergence Theorem for the finite element approximation). Let $T>0, H_{\mathrm{ext}} \geq 0$ and $\omega_{0} \in$ $\mathrm{L}^{\infty}(\Omega), \omega_{0} \geq 0$ a.e. in $\Omega$ be given. Then, as $h \rightarrow 0$, there exists a subsequence $\left\{h_{k}\right\}$ such that

$$
\begin{aligned}
u_{h_{k}} & \rightarrow u \text { strongly in } \mathrm{L}^{2}\left(0, T ; \mathrm{L}^{2}(\Omega)\right) \text { and weakly* in } \mathrm{L}^{\infty}\left(\Omega_{T}\right), \\
\omega_{h_{k}} & \rightarrow \omega \text { weakly in } \mathrm{H}^{1}\left(0, T ; \mathrm{H}^{-1}(\Omega)\right) \text { and weakly in } \mathrm{L}^{\infty}\left(\Omega_{T}\right), \\
\mathbf{p}_{h_{k}} & \rightarrow \mathbf{p} \text { strongly in } \mathrm{L}^{2}\left(0, T ; \mathrm{L}^{2}(\Omega)\right), \\
\operatorname{div} \mathbf{p}_{h_{k}} & \rightarrow \operatorname{div} \mathbf{p} \text { weakly in } \mathrm{L}^{2}\left(0, T ; \mathrm{L}^{2}(\Omega)\right),
\end{aligned}
$$

where $(u, \omega)$ is a weak solution of (2.3-2.5) and $\mathbf{p}=-\nabla u$.

The proof of Theorem 4.1 involves standard steps as similar to that in [8] and it is presented in detail later in Section 6. The main ingredients are the estimates for the Raviart-Thomas elements without resorting to the discrete maximum principle.

Clearly, Theorem 4.1 also implies that if the weak solution for (2.3-2.4) is unique, then the weak limit in the above theorem is independent of the choice of the subsequence. This in turn implies that, in this case, the whole sequence $\left\{\left(u_{h}, \omega_{h}\right)\right\}$ is convergent to the unique weak solution of (2.3-2.4). For more discussion on the existence and uniqueness of the weak solutions, we refer to $[17,22]$.

\section{Estimates FOR THE NUMERICAL SOLUtion}

We start with the maximum norm estimates.

Lemma 5.1. Given $H_{\mathrm{ext}} \geq 0$ and non-negative $\omega_{0} \in \mathrm{L}^{\infty}(\Omega)$, let

$$
M(t)=\left\|\omega_{h}(t)\right\|_{L^{\infty}(\Omega)}, \quad L(t)=\max \left(\left\|u_{h}\right\|_{L^{\infty}(\Omega \times[0, t])},\left\|\omega_{0}\right\|_{L^{\infty}(\Omega)}\right) .
$$

Then for a.e. $t \in(0, T)$,

$$
\begin{aligned}
& \omega_{h}(t) \geq 0, \\
& M(t) \leq L(t) .
\end{aligned}
$$

Proof. The proof of (5.1) is similar to the argument given in [8] by the fact that $\omega_{h}(0) \geq 0$. We first notice that (3.1) can be rewritten in the vector form as

$$
\frac{\mathrm{d}}{\mathrm{d} t} \vec{\omega}(t)+D(t) \vec{\omega}(t)-B(t) \vec{\omega}(t)=\overrightarrow{0}
$$

where $D(t)$ has a diagonal matrix form containing nonnegative entries, $B(t)$ has zeros on the diagonal and nonnegative off-diagonal entries. Since the entries of $D(t)$ is continuous in time, we may take a large enough 
constant $\lambda>0$ such that the entries of $D(t)$ is bounded by $\lambda$ for all $t \in[0, T]$. Then, by taking integration factor, we get

$$
\frac{\mathrm{d}}{\mathrm{d} t}\left(e^{\lambda t} \vec{\omega}\right)=(\lambda I-D(t)+B(t)) e^{\lambda t} \vec{\omega}
$$

where $I$ is the identity matrix. Now if $\vec{\omega}=\left(\omega_{K}\right)_{K \in \Sigma_{h}}$, multiplying the above equality by $e^{\lambda t} \vec{\eta}$ with $\vec{\eta}=$ $\left(\min \left(0, \omega_{K}\right)\right)_{K \in \Sigma_{h}}$, we deduce easily that $\vec{\eta}=\overrightarrow{0}$, which implies $\omega_{h}(t) \geq 0$ for any $t \in[0, T]$ by the fact that $\omega_{h}(0) \geq 0$.

To show (5.2), we first remark that since $\omega_{h}(t), u_{h}(t)$ are the solutions of the ODEs (3.1)-(3.4), they satisfy $\omega_{h} \in C\left(0, T ; Q_{h}\right)$ and $u_{h} \in C\left(0, T ; Q_{h}\right), M(t), L(t)$ are continuous functions in time. For any $\varepsilon>0$, since $M(0)<L(0)+\varepsilon$, we know that $M(t) \leq L(t)+\varepsilon$ at least for small $t$ which may depend on $\varepsilon$. Now we prove $M(t) \leq L(t)+\varepsilon$ for all time.

By (3.2),

$$
\begin{aligned}
\int_{\partial K} \mathbf{p}_{h} \cdot \mathbf{n} \tilde{\omega}_{h} \mathrm{~d} \gamma & =\int_{\partial K} \mathbf{p}_{h} \cdot \mathbf{n}\left(\tilde{\omega}_{h}-\omega_{h}\right) \mathrm{d} \gamma+\int_{\partial K} \mathbf{p}_{h} \cdot \mathbf{n} \omega_{h} \mathrm{~d} \gamma \\
& =\int_{\partial K_{-}} \mathbf{p}_{h} \cdot \mathbf{n}\left(\omega_{h}^{-}-\omega_{h}\right) \mathrm{d} \gamma+\left(\operatorname{div} \mathbf{p}_{h}, \omega_{h}\right)_{K} \\
& =\int_{\partial K_{-}} \mathbf{p}_{h} \cdot \mathbf{n}\left(\omega_{h}^{-}-\omega_{h}\right) \mathrm{d} \gamma+\left(\omega_{h}-u_{h}, \omega_{h}\right)_{K}
\end{aligned}
$$

Let

$$
\left.\omega_{h}(t)\right|_{K^{*}}=M(t)=\max _{K \in \Sigma_{h}}\left\{\left.\omega_{h}(t)\right|_{K}\right\},
$$

then, we have

$$
\int_{\partial K_{-}^{*}} \mathbf{p}_{h} \cdot \mathbf{n}\left(\omega_{h}^{-}-\omega_{h}\right) \mathrm{d} \gamma \geq 0
$$

Thus, by (3.1), we have

$$
\frac{\mathrm{d} \omega_{h}}{\mathrm{~d} t}+\left(\omega_{h}-u_{h}\right) \omega_{h} \leq 0 \quad \text { on } K^{*}
$$

Suppose that $M(t) \leq L(t)+\varepsilon$ was not true for all $t>0$. Let $t^{*}$ be the smallest time such that $M\left(t^{*}\right)=L\left(t^{*}\right)+\varepsilon$ and $M(t)<L(t)+\varepsilon$ for all $0 \leq t<t^{*}$. Thus $M\left(t^{*}\right)>L\left(t^{*}\right)$ and, consequently, $\omega_{h}\left(t^{*}\right)>u_{h}\left(t^{*}\right)$ on $K^{*}$, so

$$
\frac{\mathrm{d} \omega_{h}}{\mathrm{~d} t}\left(t^{*}\right)<0 \quad \text { on } K^{*}
$$

which implies that $M\left(t^{*}\right) \leq M\left(t^{\prime}\right)$ for some $t^{\prime}<t$. But $M\left(t^{*}\right)=L\left(t^{*}\right)+\varepsilon$ and $M\left(t^{\prime}\right)<L\left(t^{\prime}\right)+\varepsilon \leq L\left(t^{*}\right)+\varepsilon$, we get $L\left(t^{*}\right)<L\left(t^{*}\right)$, a contradiction! Therefore, $M(t) \leq L(t)+\varepsilon$ for any time $t>0$ and any $\varepsilon>0$. By letting $\varepsilon \rightarrow 0$, we obtain the desired estimate (5.2) for any $t>0$.

Unlike the scheme in [8], the Raviart-Thomas finite element approximations do not enjoy a discrete maximum principle in general. To obtain the energy bound on $\omega_{h}$, we first consider 
Lemma 5.2. Let $M_{1}(t)=\left\|\omega_{h}\right\|_{\mathrm{L}^{1}(\Omega)}$, then $M_{1}(t)$ is non-increasing in time. Thus, $M_{1}(t) \leq M_{1}(0)$.

Proof. Taking $\eta_{h}=1$ in (3.1), and noticing the cancellation of terms on the inter-element edges and using (5.1), we get

$$
\begin{aligned}
\frac{\mathrm{d}}{\mathrm{d} t}\left\|\omega_{h}\right\|_{\mathrm{L}^{1}(\Omega)} & =-\sum_{K \in \Sigma_{h}} \int_{\partial K}\left(\mathbf{p}_{h} \cdot \mathbf{n}\right) \tilde{\omega}_{h} \mathrm{~d} \gamma \\
& =-\sum_{K \in \Sigma_{h}} \int_{\partial K_{+}}\left(\mathbf{p}_{h} \cdot \mathbf{n}\right) \omega_{h}^{+} \mathrm{d} \gamma-\sum_{K \in \Sigma_{h}} \int_{\partial K_{-}}\left(\mathbf{p}_{h} \cdot \mathbf{n}\right) \omega_{h}^{-} \mathrm{d} \gamma \\
& =-\sum_{K \in \Sigma_{h}} \int_{\partial K_{+} \cap \Gamma}\left(\mathbf{p}_{h} \cdot \mathbf{n}\right) \omega_{h}^{+} \mathrm{d} \gamma \\
& \leq 0 .
\end{aligned}
$$

This proves the lemma.

Before we proceed to the derivation of energy estimates, let us recall some technical results concerning the Raviart-Thomas element.

Lemma 5.3. $[2,21]$ For any given $f \in \mathrm{L}^{2}(\Omega)$, let $\varphi \in \mathrm{H}_{0}^{1}(\Omega)$ be the solution of

$$
-\Delta \varphi+\varphi=f
$$

and $\left(\varphi_{h}, \boldsymbol{\theta}_{h}\right) \in Q_{h} \times P_{h}$ be the approximate solution of $(\varphi,-\nabla \varphi)$ using the mixed finite element formulation:

$$
\begin{aligned}
& \left(\operatorname{div} \boldsymbol{\theta}_{h}, v_{h}\right)+\left(\varphi_{h}, v_{h}\right)=\left(f, v_{h}\right) \quad \forall v_{h} \in Q_{h} \\
& \left(\boldsymbol{\theta}_{h}, \mathbf{q}_{h}\right)-\left(\varphi_{h}, \operatorname{div} \mathbf{q}_{h}\right)=0 \quad \forall \mathbf{q}_{h} \in P_{h}
\end{aligned}
$$

Then, for $h$ small, there exists a constant $c>0$ such that

$$
\left\|\varphi-\varphi_{h}\right\|_{\mathrm{L}^{2}(\Omega)}+\left\|\boldsymbol{\theta}_{h}-(-\nabla \varphi)\right\|_{\mathrm{L}^{2}(\Omega)} \leq \operatorname{ch}\|f\|_{\mathrm{L}^{2}(\Omega)}
$$

and, consequently, by the triangle inequality

$$
\left\|\boldsymbol{\theta}_{h}\right\|_{\mathrm{L}^{2}(\Omega)}+\left\|\varphi_{h}\right\|_{\mathrm{L}^{2}(\Omega)} \leq \operatorname{ch}\|f\|_{\mathrm{L}^{2}(\Omega)}+c\|f\|_{\mathrm{H}^{-1}(\Omega)} \text {. }
$$

We refer to $[2,21]$ for detailed proof of the above lemma. Note that throughout the paper, we use $c>0$ to denote any generic constant that is independent of the discretization parameters such as the spatial mesh size $h$ as well as $\tau$, the time step size used in the fully discrete approximation.

Lemma 5.4. There exists a constant $c>0$ independent of $h$ such that, for a.e. $t \in(0, T)$,

$$
\left\|u_{h}\right\|_{\mathrm{L}^{\infty}(\Omega)} \leq c\left\|\omega_{h}\right\|_{\mathrm{L}^{2}(\Omega)}+c .
$$

Proof. Define $u_{h}^{*}$ to be the solution of the problem

$$
\begin{aligned}
-\Delta u_{h}^{*}+u_{h}^{*} & =\omega_{h} \quad \text { in } \Omega, \\
u_{h}^{*} & =H_{\text {ext }} \quad \text { on } \Gamma .
\end{aligned}
$$

Then from the error estimate in Lemma 5.3, we know that

$$
\left\|u_{h}-u_{h}^{*}\right\|_{\mathrm{L}^{2}(\Omega)} \leq \operatorname{ch}\left\|\omega_{h}-H_{\mathrm{ext}}\right\|_{\mathrm{L}^{2}(\Omega)}
$$


By using the elliptic regularity theory on convex polygons [15] to (5.8-5.9) we know that

$$
\left\|u_{h}^{*}\right\|_{H^{2}(\Omega)} \leq c\left\|\omega_{h}\right\|_{L^{2}(\Omega)}+c
$$

Now denote by $I_{h}: \mathrm{L}^{2}(\Omega) \rightarrow Q_{h}$ the $\mathrm{L}^{2}$ projection operator, then the following error estimate is well-known [1]

$$
\left\|\varphi-I_{h} \varphi\right\|_{\mathrm{L}^{2}(\Omega)} \leq \operatorname{ch}\|\varphi\|_{\mathrm{H}^{1}(\Omega)} \quad \forall \varphi \in \mathrm{H}^{1}(\Omega)
$$

Then by using the above two estimates and the inverse inequality [1] we get

$$
\begin{aligned}
\left\|u_{h}-I_{h} u_{h}^{*}\right\|_{\mathrm{L}^{\infty}(\Omega)} & \leq c \mathrm{H}^{-1}\left\|u_{h}-I_{h} u_{h}^{*}\right\|_{\mathrm{L}^{2}(\Omega)} \\
& \leq c \mathrm{H}^{-1}\left\|u_{h}-u_{h}^{*}\right\|_{\mathrm{L}^{2}(\Omega)}+c \mathrm{H}^{-1}\left\|u_{h}^{*}-I_{h} u_{h}^{*}\right\|_{\mathrm{L}^{2}(\Omega)} \\
& \leq c\left\|\omega_{h}-H_{\mathrm{ext}}\right\|_{\mathrm{L}^{2}(\Omega)}+c\left\|u_{h}^{*}\right\|_{\mathrm{H}^{1}(\Omega)} \\
& \leq c\left\|\omega_{h}\right\|_{\mathrm{L}^{2}(\Omega)}+c .
\end{aligned}
$$

This proves the desired bound of $u_{h}$ by Sobolev embedding theorem and (5.10).

Lemma 5.5. There exists a constant $c>0$ independent of $h$, such that

$$
\sup _{0 \leq t \leq T}\left\|\omega_{h}\right\|_{L^{2}(\Omega)}^{2}+\sum_{K \in \Sigma_{h}} \int_{0}^{T} \int_{\partial K_{-}}\left|\mathbf{p}_{h} \cdot \mathbf{n}\right|\left[\omega_{h}\right]^{2} \mathrm{~d} \gamma \leq c
$$

Proof. Let us take $\eta_{h}=\omega_{h}$ in (3.1), then

$$
\frac{1}{2} \frac{\mathrm{d}}{\mathrm{d} t}\left\|\omega_{h}\right\|_{\mathrm{L}^{2}(\Omega)}^{2}+\sum_{K \in \Sigma_{h}} \int_{\partial K} \mathbf{p}_{h} \cdot \mathbf{n} \tilde{\omega}_{h} \omega_{h} \mathrm{~d} \gamma=0
$$

On the boundary $\partial K$, we have

$$
\int_{\partial K} \mathbf{p}_{h} \cdot \mathbf{n} \tilde{\omega}_{h} \omega_{h} \mathrm{~d} \gamma=\int_{\partial K} \mathbf{p}_{h} \cdot \mathbf{n}\left(\tilde{\omega}_{h} \omega_{h}-\frac{1}{2} \omega_{h}^{2}\right) \mathrm{d} \gamma+\frac{1}{2} \int_{\partial K} \mathbf{p}_{h} \cdot \mathbf{n} \omega_{h}^{2} \mathrm{~d} \gamma
$$

Noticing that

$$
\sum_{K \in \Sigma_{h}} \int_{\partial K_{+}} \mathbf{p}_{h} \cdot \mathbf{n} \omega_{h}^{+} \omega_{h}^{+} \mathrm{d} \gamma=-\sum_{K \in \Sigma_{h}} \int_{\partial K_{-}} \mathbf{p}_{h} \cdot \mathbf{n} \omega_{h}^{-} \omega_{h}^{-} \mathrm{d} \gamma+\int_{\Gamma_{h}^{+}} \mathbf{p}_{h} \cdot \mathbf{n} \omega_{h}^{2} \mathrm{~d} \gamma
$$


we get,

$$
\begin{aligned}
& \sum_{K \in \Sigma_{h}} \int_{\partial K} \mathbf{p}_{h} \cdot \mathbf{n}\left(\tilde{\omega}_{h} \omega_{h}-\frac{1}{2} \omega_{h}^{2}\right) \mathrm{d} \gamma \\
& =\frac{1}{2} \sum_{K \in \Sigma_{h}} \int_{\partial K_{+}} \mathbf{p}_{h} \cdot \mathbf{n} \omega_{h}^{+} \omega_{h}^{+} \mathrm{d} \gamma+\sum_{K \in \Sigma_{h}} \int_{\partial K_{-}} \mathbf{p}_{h} \cdot \mathbf{n}\left(\omega_{h}^{-} \omega_{h}^{+}-\frac{1}{2}\left(\omega_{h}^{+}\right)^{2}\right) \mathrm{d} \gamma \\
& =-\frac{1}{2} \sum_{K \in \Sigma_{h}} \int_{\partial K_{-}} \mathbf{p}_{h} \cdot \mathbf{n}\left(\left(\omega_{h}^{-}\right)^{2}-2 \omega_{h}^{-} \omega_{h}^{+}+\left(\omega_{h}^{+}\right)^{2}\right) \mathrm{d} \gamma+\frac{1}{2} \int_{\Gamma_{h}^{+}}\left|\mathbf{p}_{h} \cdot \mathbf{n}\right| \omega_{h}^{2} \mathrm{~d} \gamma \\
& =-\frac{1}{2} \sum_{K \in \Sigma_{h}} \int_{\partial K_{-}} \mathbf{p}_{h} \cdot \mathbf{n}\left[\omega_{h}\right]^{2} \mathrm{~d} \gamma+\frac{1}{2} \int_{\Gamma_{h}^{+}}\left|\mathbf{p}_{h} \cdot \mathbf{n}\right| \omega_{h}^{2} \mathrm{~d} \gamma \\
& =\frac{1}{2} \sum_{K \in \Sigma_{h}} \int_{\partial K_{-}}\left|\mathbf{p}_{h} \cdot \mathbf{n}\right|\left[\omega_{h}\right]^{2} \mathrm{~d} \gamma+\frac{1}{2} \int_{\Gamma_{h}^{+}}\left|\mathbf{p}_{h} \cdot \mathbf{n}\right| \omega_{h}^{2} \mathrm{~d} \gamma
\end{aligned}
$$

On the other hand, since $\omega_{h} \geq 0$ by Lemma 5.1 , we have

$$
\begin{aligned}
\sum_{K \in \Sigma_{h}} \int_{\partial K} \mathbf{p}_{h} \cdot \mathbf{n} \omega_{h}^{2} \mathrm{~d} \gamma & =\sum_{K \in \Sigma_{h}} \int_{K} \operatorname{div} \mathbf{p}_{h} \omega_{h}^{2} \mathrm{~d} \mathbf{x} \\
& =\left(\omega_{h}-u_{h}, \omega_{h}^{2}\right) \\
& \geq-\left(u_{h}, \omega_{h}^{2}\right) \\
& \geq-M_{1}(0)\left\|\omega_{h}\right\|_{\mathrm{L}^{\infty}(\Omega)}\left\|u_{h}\right\|_{\mathrm{L}^{\infty}(\Omega)} \\
& \geq-c \max _{0 \leq s \leq t}\left\|\omega_{h}(s)\right\|_{\mathrm{L}^{2}(\Omega)}^{2}-c,
\end{aligned}
$$

where in the last two inequalities we have used Lemmas 5.1, 5.2 and 5.4. Thus

$$
\frac{1}{2} \frac{\mathrm{d}}{\mathrm{d} t}\left\|\omega_{h}\right\|_{\mathrm{L}^{2}(\Omega)}^{2}+\frac{1}{2} \sum_{K \in \Sigma_{h}} \int_{\partial K_{-}}\left|\mathbf{p}_{h} \cdot \mathbf{n}\right|\left[\omega_{h}\right]^{2} \mathrm{~d} \gamma \leq c \max _{0 \leq s \leq t}\left\|\omega_{h}(s)\right\|_{\mathrm{L}^{2}(\Omega)}^{2}+c .
$$

Using Gronwall inequality, we get the desired estimate.

From Lemmas 5.1, 5.4 and 5.5 we obtain easily the following uniform bound of $u_{h}$ and $\omega_{h}$.

Corollary 5.6. There exists a constant $c>0$ such that

$$
\left\|u_{h}\right\|_{\mathrm{L}^{\infty}(\Omega)}+\left\|\omega_{h}\right\|_{\mathrm{L}^{\infty}(\Omega)} \leq c
$$

uniformly with respect to $h$.

Next, let us state a simple estimate on $\mathbf{p}_{h}$.

Lemma 5.7. There exists a constant $c>0$ such that for a.e. $t \in(0, T)$,

$$
\left\|\operatorname{div} \mathbf{p}_{h}\right\|_{L^{2}(\Omega)}^{2}+\left\|\mathbf{p}_{h}\right\|_{L^{2}(\Omega)}^{2} \leq c
$$

Proof. Though there are a number of different proofs, we simply verify it by taking $v_{h}=u_{h}-H_{\text {ext }}$ in (3.2) and $\mathbf{q}_{h}=\mathbf{p}_{h}$ in (3.3) and applying the earlier maximum norm estimates. First, we have

$$
\begin{aligned}
& \left\|\mathbf{p}_{h}\right\|_{\mathrm{L}^{2}(\Omega)}^{2}+\left\|u_{h}-H_{\mathrm{ext}}\right\|_{\mathrm{L}^{2}(\Omega)}^{2}=\left(\omega_{h}-H_{\mathrm{ext}}, u_{h}-H_{\mathrm{ext}}\right) \\
& \quad \leq \frac{1}{2}\left\|u_{h}(t)-H_{\mathrm{ext}}\right\|_{\mathrm{L}^{2}(\Omega)}^{2}+c
\end{aligned}
$$


for some generic constant $c>0$, independent of $h$. Then, by taking $v_{h}=\operatorname{div} \mathbf{p}_{h}$ in (3.2), we get

$$
\begin{aligned}
\left\|\operatorname{div} \mathbf{p}_{h}\right\|_{L^{2}(\Omega)}^{2} & =\left(\omega_{h}-u_{h}, \operatorname{div} \mathbf{p}_{h}\right) \\
& \leq\left(\left\|\omega_{h}\right\|_{L^{2}(\Omega)}+\left\|u_{h}\right\|_{L^{2}(\Omega)}\right)\left\|\operatorname{div} \mathbf{p}_{h}\right\|_{L^{2}(\Omega)} \leq c\left\|\operatorname{div} \mathbf{p}_{h}\right\|_{L^{2}(\Omega)}
\end{aligned}
$$

for some generic constant $c>0$, independent of $h$. This proves the lemma.

Following from Lemma 5.7 and Lemma 5.3, we can get some uniform bound on the time derivatives of the discrete solutions.

Lemma 5.8. There exists a constant $c>0$ such that

$$
\begin{aligned}
& \left\|\omega_{h t}\right\|_{\mathrm{L}^{\infty}\left(0, T ; \mathrm{H}^{-1}(\Omega)\right)} \leq c \\
& \left\|\omega_{h t}\right\|_{\mathrm{L}^{\infty}\left(0, T ; \mathrm{L}^{2}(\Omega)\right)} \leq c h^{-1}
\end{aligned}
$$

uniformly with respect to $h$.

Proof. Let $\varphi \in \mathrm{C}_{0}^{\infty}(\Omega)$, and $\varphi_{h}$ be its $\mathrm{L}^{2}$ projection in $Q_{h}$. With the notation $h_{K}=\operatorname{diam}(K)$ for any $K \in \Sigma_{h}$, we consider

$$
\begin{aligned}
& \left|\left(\omega_{h t}, \varphi\right)\right|=\left|\left(\omega_{h t}, \varphi_{h}\right)\right| \\
& =\left|\sum_{K \in \Sigma_{h}} \int_{\partial K} \mathbf{p}_{h} \cdot \mathbf{n} \tilde{\omega}_{h} \varphi_{h} \mathrm{~d} \gamma\right| \\
& =\left|\sum_{K \in \Sigma_{h}} \int_{\partial K_{+}} \mathbf{p}_{h} \cdot \mathbf{n} \omega_{h}^{+} \varphi_{h}^{+} \mathrm{d} \gamma+\sum_{K \in \Sigma_{h}} \int_{\partial K_{-}} \mathbf{p}_{h} \cdot \mathbf{n} \omega_{h}^{-} \varphi_{h}^{+} \mathrm{d} \gamma\right| \\
& \leq\left|\sum_{K \in \Sigma_{h}} \int_{\partial K_{+}} \mathbf{p}_{h} \cdot \mathbf{n} \omega_{h}^{+}\left[\varphi_{h}\right] \mathrm{d} \gamma\right| \\
& \quad \leq c\left(\sum_{K \in \Sigma_{h}} h_{K} \int_{\partial K_{+}}\left|\mathbf{p}_{h} \cdot \mathbf{n}\right|^{2} \mathrm{~d} \gamma\right)^{\frac{1}{2}}\left(\sum_{K \in \Sigma_{h}} h_{K}^{-1} \int_{\partial K_{+}}\left|\left[\varphi_{h}\right]\right|^{2} \mathrm{~d} \gamma\right)^{\frac{1}{2}}
\end{aligned}
$$

for some constant $c>0$ which depends only on the uniform estimate for $\omega_{h}$. Using a local inverse estimate

$$
\left\|\mathbf{p}_{h}\right\|_{L^{2}(\partial K)} \leq c h_{K}^{-1 / 2}\left\|\mathbf{p}_{h}\right\|_{L^{2}(K)}
$$

we have

$$
\sum_{K \in \Sigma_{h}} h_{K} \int_{\partial K_{+}}\left|\mathbf{p}_{h} \cdot \mathbf{n}\right|^{2} \mathrm{~d} \gamma \leq c\left\|\mathbf{p}_{h}\right\|_{L^{2}(\Omega)}^{2},
$$

for some constant $c>0$. By the approximation properties of the $\mathrm{L}^{2}$ projection, we get

$$
\int_{\partial K}\left|\varphi_{h}-\varphi\right|^{2} \mathrm{~d} \gamma \leq c h_{K}\|\varphi\|_{\mathrm{H}^{1}(K)}^{2}
$$

Thus

$$
\left|\left(\omega_{h t}, \varphi\right)\right| \leq c\|\varphi\|_{\mathrm{H}^{1}(\Omega)}
$$


for some generic constant $c>0$. This gives the bound (5.12) in the lemma. The bound (5.13) can be proved similarly by noticing that

$$
\left(\sum_{K \in \Sigma_{h}} h_{K}^{-1} \int_{\partial K_{+}}\left|\left[\varphi_{h}\right]\right|^{2} \mathrm{~d} \gamma\right)^{\frac{1}{2}} \leq c h^{-1}\left\|\varphi_{h}\right\|_{\mathrm{L}^{2}(\Omega)} \leq c h^{-1}\|\varphi\|_{\mathrm{L}^{2}(\Omega)} .
$$

This completes the proof.

Now, the equations (3.2-3.3) implies that

$$
\begin{gathered}
\left(\operatorname{div} \mathbf{p}_{h t}, v_{h}\right)+\left(u_{h t}-\omega_{h t}, v_{h}\right)=0, \quad \forall v_{h} \in Q_{h}, \\
\left(\mathbf{p}_{h t}, \mathbf{q}_{h}\right)-\left(u_{h t}, \operatorname{div} \mathbf{q}_{h}\right)=0 \quad \forall \mathbf{q}_{h} \in P_{h} .
\end{gathered}
$$

Thus, we see that $\left(u_{h t}, \mathbf{p}_{h t}\right)$ is the finite element approximation of the solution $\left(\varphi_{h}^{*},-\nabla \varphi_{h}^{*}\right)$ of the equation

$$
-\Delta \varphi_{h}^{*}+\varphi_{h}^{*}=\omega_{h t} \quad \text { in } \Omega
$$

with homogeneous Dirichlet boundary condition.

By applying Lemma 5.3 and Lemma 5.8, we get

Corollary 5.9. There exists a generic constant $c>0$, such that

$$
\left\|\mathbf{p}_{h t}\right\|_{\mathrm{L}^{2}(\Omega)}+\left\|u_{h t}\right\|_{\mathrm{L}^{2}(\Omega)} \leq c
$$

uniformly with respect to $h$.

Combining the above estimates, we get

Corollary 5.10. For given $T>0, H_{\mathrm{ext}} \geq 0$ and given $\omega_{0} \in \mathrm{L}^{\infty}(\Omega)$ such that $\omega_{0} \geq 0$ a.e. in $\Omega$, there exists some constant $c>0$ such that

$$
\begin{aligned}
& \left\|u_{h t}\right\|_{\mathrm{L}^{\infty}\left(0, T ; \mathrm{L}^{2}(\Omega)\right)}+\left\|u_{h}\right\|_{\mathrm{L}^{\infty}\left(\Omega_{T}\right)} \leq c, \\
& \left\|\omega_{h t}\right\|_{\mathrm{L}^{\infty}\left(0, T ; \mathrm{H}^{-1}(\Omega)\right)}+\left\|\omega_{h}\right\|_{\mathrm{L}^{\infty}\left(\Omega_{T}\right)} \leq c, \\
& \left\|\mathbf{p}_{h t}\right\|_{\mathrm{L}^{\infty}\left(0, T ; \mathrm{L}^{2}(\Omega)\right)}+\left\|\mathbf{p}_{h}\right\|_{\mathrm{L}^{\infty}(0, T ; H(\operatorname{div} ; \Omega))} \leq c,
\end{aligned}
$$

uniformly with respect to $h$.

\section{Proof of the Main CONVERGEnCE THEOREM}

Based on the uniform bound on the discrete solutions, we first extract weakly or weakly* convergent sequences.

Lemma 6.1. Let $T>0, H_{\mathrm{ext}} \geq 0$ and $\omega_{0} \in \mathrm{L}^{\infty}(\Omega)$ such that $\omega_{0} \geq 0$ a.e. in $\Omega$ be given. Then, any subsequence of $\left\{\omega_{h}, u_{h}, \mathbf{p}_{h}\right\}$ has a subsequence $\left\{\omega_{h_{k}}, u_{h_{k}}, \mathbf{p}_{h_{k}}\right\}$ such that as $h_{k} \rightarrow 0$,

$$
\begin{aligned}
u_{h_{k}} & \rightarrow u \text { strongly in } \mathrm{L}^{2}\left(0, T ; \mathrm{L}^{2}(\Omega)\right) \text { and weakly }{ }^{*} \text { in } \mathrm{L}^{\infty}\left(\Omega_{T}\right), \\
\omega_{h_{k}} & \rightarrow \omega \text { weakly in } \mathrm{H}^{1}\left(0, T ; \mathrm{H}^{-1}(\Omega)\right) \text { and weakly* in } \mathrm{L}^{\infty}\left(\Omega_{T}\right), \\
\mathbf{p}_{h_{k}} & \rightarrow \mathbf{p} \text { strongly in } \mathrm{L}^{2}\left(0, T ; \mathrm{L}^{2}(\Omega)\right), \\
\operatorname{div} \mathbf{p}_{h_{k}} & \rightarrow \text { div } \mathbf{p} \text { weakly in } \mathrm{L}^{2}\left(0, T ; \mathrm{L}^{2}(\Omega)\right),
\end{aligned}
$$

for some limit $\{\omega, u, \mathbf{p}\}$ in the appropriate spaces. 
Proof. The existence of the weak or weak* limit $\{\omega, u, \mathbf{p}\}$ and the weak or weak* convergent subsequence $\left\{\omega_{h_{k}}, u_{h_{k}}, \mathbf{p}_{h_{k}}\right\}$ follows from the uniform estimates in Corollary 5.10. We only need to prove the strong convergence in (6.1) and (6.3). Consider the auxiliary problem (5.8-5.9), we get $\left\{u_{h_{k}}^{*}\right\}$ is uniformly bounded in $\mathrm{H}^{1}\left(0, T ; \mathrm{H}^{1}(\Omega)\right) \cap \mathrm{L}^{2}\left(0, T ; H^{2}(\Omega)\right)$, after possibly extracting another subsequence, we may use the compact embedding results [23] to get $\left\{u_{h_{k}}^{*}\right\}$ converges weakly in $\mathrm{H}^{1}\left(0, T ; \mathrm{H}^{1}(\Omega)\right) \cap \mathrm{L}^{2}\left(0, T ; H^{2}(\Omega)\right)$ to some limit $u$ and the convergence is strong in $\mathrm{L}^{2}\left(0, T, \mathrm{H}^{1}(\Omega)\right)$, which in turn implies that $\left\{-\nabla u_{h_{k}}^{*}\right\}$ converges strongly in $\mathrm{L}^{2}(0, T$; $\left.\mathrm{L}^{2}(\Omega)\right)$ to $\mathbf{p}=-\nabla u$. Then, using the error estimate for the problem (5.8-5.9), we get the strong convergence of $u_{h_{k}}$ to $u$ in $\mathrm{L}^{2}\left(0, T ; \mathrm{L}^{2}(\Omega)\right)$ and $\mathbf{p}_{h_{k}}$ to $\mathbf{p}$ in $\mathrm{L}^{2}\left(0, T ; \mathrm{L}^{2}(\Omega)\right)$.

As a consequence, we can get easily from the equations (3.2-3.3) that the weak limit satisfies the weak form (2.4)-(2.5).

Proposition 6.2. The limit $\{u, \mathbf{p}, \omega\}$ satisfies Equations (2.4)-(2.5).

Let us now check the equation (2.3).

Proposition 6.3. The limit $\{u, \mathbf{p}, \omega\}$ satisfies Equation (2.3).

Proof. For simplicity, we avoid the use of the subscripts for the sequence $\left\{h_{k}\right\}$. First, for $\phi \in \mathrm{C}_{0}^{\infty}(\Omega \times[0, T))$, as $h \rightarrow 0$, we have

$$
\begin{aligned}
\int_{0}^{T}\left(\omega_{h t}, \phi\right) \mathrm{d} t & =-\int_{0}^{T}\left(\omega_{h}, \phi_{t}\right) \mathrm{d} t+\left(\omega_{h, 0}, \phi(\cdot, 0)\right) \\
& \rightarrow-\int_{0}^{T}\left(\omega, \phi_{t}\right) \mathrm{d} t+\left(\omega_{0}, \phi(\cdot, 0)\right)
\end{aligned}
$$

Meanwhile, let $\phi_{h}$ be the piecewise constant $\mathrm{L}^{2}$ projection of $\phi$ in $Q_{h}$,

$$
\begin{aligned}
\left(\omega_{h t}, \phi\right) & =\left(\omega_{h t}, \phi_{h}\right) \\
& =-\sum_{K \in \Sigma_{h}} \int_{\partial K} \mathbf{p}_{h} \cdot \mathbf{n} \tilde{\omega}_{h} \phi_{h} \mathrm{~d} \gamma \\
& =\sum_{K \in \Sigma_{h}} \int_{\partial K} \mathbf{p}_{h} \cdot \mathbf{n}\left(\omega_{h}-\tilde{\omega}_{h}\right) \phi_{h} \mathrm{~d} \gamma-\sum_{K \in \Sigma_{h}} \int_{\partial K} \mathbf{p}_{h} \cdot \mathbf{n} \omega_{h} \phi_{h} \mathrm{~d} \gamma .
\end{aligned}
$$

We also have

$$
\begin{aligned}
\sum_{K \in \Sigma_{h}} \int_{\partial K} \mathbf{p}_{h} \cdot \mathbf{n} \omega_{h} \phi_{h} \mathrm{~d} \gamma=\sum_{K \in \Sigma_{h}} \int_{K} \operatorname{div} \mathbf{p}_{h} \omega_{h} \phi_{h} \mathrm{~d} \tau=\sum_{K \in \Sigma_{h}} \int_{K} \operatorname{div} \mathbf{p}_{h} \omega_{h} \phi \mathrm{d} \tau \\
=-\sum_{K \in \Sigma_{h}} \int_{K} \mathbf{p}_{h} \omega_{h} \nabla \phi \mathrm{d} \mathbf{x}+\sum_{K \in \Sigma_{h}} \int_{\partial K} \mathbf{p}_{h} \cdot \mathbf{n} \omega_{h} \phi \mathrm{d} \gamma
\end{aligned}
$$

and

Moreover,

$$
\sum_{K \in \Sigma_{h}} \int_{\partial K} \mathbf{p}_{h} \cdot \mathbf{n} \omega_{h} \phi \mathrm{d} \gamma=\sum_{K \in \Sigma_{h}} \int_{\partial K_{-}} \mathbf{p}_{h} \cdot \mathbf{n}\left[\omega_{h}\right] \phi \mathrm{d} \gamma
$$

We thus get

$$
\sum_{K \in \Sigma_{h}} \int_{\partial K} \mathbf{p}_{h} \cdot \mathbf{n}\left(\omega_{h}-\tilde{\omega}_{h}\right) \phi_{h} \mathrm{~d} \gamma=\sum_{K \in \Sigma_{h}} \int_{\partial K_{-}} \mathbf{p}_{h} \cdot \mathbf{n}\left[\omega_{h}\right] \phi_{h} \mathrm{~d} \gamma
$$

$$
\left(\omega_{h t}, \phi\right)=\left(\mathbf{p}_{h} \omega_{h}, \nabla \phi\right)-\sum_{K \in \Sigma_{h}} \int_{\partial K_{-}} \mathbf{p}_{h} \cdot \mathbf{n}\left[\omega_{h}\right]\left(\phi-\phi_{h}\right) \mathrm{d} \gamma
$$


On the other hand, by Lemma 5.5 and the finite element interpolation estimate

$$
\left\|\phi-\phi_{h}\right\|_{L^{\infty}(K)} \leq c h_{K}\|\phi\|_{W^{1, \infty}(K)} \leq c h_{K}
$$

we have

$$
\begin{aligned}
& \sum_{K \in \Sigma_{h}} \int_{\partial K_{-}}\left|\mathbf{p}_{h} \cdot \mathbf{n}\left[\omega_{h}\right]\left(\phi-\phi_{h}\right)\right| \mathrm{d} \gamma \\
& \leq\left(\sum_{K \in \Sigma_{h}} \int_{\partial K_{-}}\left|\mathbf{p}_{h} \cdot \mathbf{n}\right|\left[\omega_{h}\right]^{2} \mathrm{~d} \gamma\right)^{\frac{1}{2}}\left(\sum_{K \in \Sigma_{h}} \int_{\partial K_{-}}\left|\mathbf{p}_{h} \cdot \mathbf{n}\right|\left(\phi-\phi_{h}\right)^{2} \mathrm{~d} \gamma\right)^{\frac{1}{2}} \\
& \leq c\left(\sum_{K \in \Sigma_{h}} h_{K}^{2} \int_{\partial K_{-}}\left|\mathbf{p}_{h} \cdot \mathbf{n}\right| \mathrm{d} \gamma\right)^{\frac{1}{2}} \\
& \leq c h^{1 / 2} \int_{\Omega}\left|\mathbf{p}_{h}\right| \mathrm{d} \mathbf{x} \rightarrow 0
\end{aligned}
$$

uniformly in $h$ and $t \in(0, T)$. So, by the weak* convergence of $\omega_{h}$ and the strong convergence of $\mathbf{p}_{h}$, we get from (6.6) that

$$
\int_{0}^{T}\left(\omega_{h t}, \phi\right) \mathrm{d} t \rightarrow \int_{0}^{T} \int_{\Omega} \omega \mathbf{p} \cdot \nabla \phi \mathrm{d} \mathbf{x} \mathrm{d} t .
$$

Combining the equations (6.5) and (6.7), we get that $(u, \omega)$ satisfies $(2.3)$.

By Propositions 6.2 and 6.3, we have completed the proof of the main theorem. Note again that if the weak solution to (2.3-2.4) is unique, then the whole sequence converges.

\section{Fully Discrete SCHEME}

In this section we discuss briefly the further discretization in time of the semi-discrete scheme (3.1-3.4) of section 3 and thus obtain a scheme which can be directly implemented on computers. For $n \geq 1$, let $\tau_{n}$ be the time step size and denote by $t^{n}=\sum_{k=1}^{n} \tau_{k}$. Let $W^{0}=\omega_{h, 0} \in Q_{h}$ be given according to (3.4). For $n \geq 1$ and given $W^{n-1} \in Q_{h}$ which is known as the approximation of $\omega$ at the $(n-1)$ th time step, we first further discretize (3.2-3.3) in time according to the following prescription

$$
\begin{aligned}
& \left(\operatorname{div} \mathbf{P}^{n}, v_{h}\right)+\left(U^{n}, v_{h}\right)=\left(W^{n-1}, v_{h}\right) \quad \forall v_{h} \in Q_{h}, \\
& \left(\mathbf{P}^{n}, \mathbf{q}_{h}\right)-\left(U^{n}-H_{\text {ext }}, \operatorname{div} \mathbf{q}_{h}\right)=0 \quad \forall \mathbf{q}_{h} \in P_{h} .
\end{aligned}
$$

It is clear that (7.1-7.2) admits a unique solution $\left(U^{n}, \mathbf{P}^{n}\right) \in Q_{h} \times P_{h}$ which we take as the approximation of $(u, p)$ at the $n$th time step. With $\mathbf{P}^{n} \in P_{h}$ being known, we can divide the boundary $\partial K$ of each element $K \in \Sigma_{h}$ into two parts

$$
\begin{array}{lll}
\partial K_{-}^{n}=\cup\left\{\gamma \subset \partial K: \mathbf{P}^{n}\left(\mathbf{x}_{\gamma}\right) \cdot \mathbf{n}<0\right\} & \text { inflow }, \\
\partial K_{+}^{n}=\cup\left\{\gamma \subset \partial K: \mathbf{P}^{n}\left(\mathbf{x}_{\gamma}\right) \cdot \mathbf{n} \geq 0\right\} & \text { outflow },
\end{array}
$$

and introduce the upwind value of any $\eta_{h} \in Q_{h}$ on $\partial K$ as

$$
\tilde{\eta}_{h}^{n}= \begin{cases}\eta_{h}^{+}\left(\text {interior trace of } \eta_{h}\right) & \text { on } \partial K_{+}^{n} \\ \eta_{h}^{-}\left(\text {exterior trace of } \eta_{h}\right) & \text { on } \partial K_{-}^{n}\end{cases}
$$


and assume $\eta_{h}^{-}=0$ on $\partial K_{-}^{n} \cap \Gamma$. Now we define the fully discrete approximation of (1.1) as follows

$$
\left(\frac{W^{n}-W^{n-1}}{\tau_{n}}, \eta_{h}\right)_{K}+\int_{\partial K} \mathbf{P}^{n} \cdot \mathbf{n} \tilde{W}^{n} \eta_{h} \mathrm{~d} \gamma=0 \quad \forall \eta_{h} \in Q_{h}, K \in \Sigma_{h} .
$$

The existence and uniqueness of $W^{n}$ is considered in the following proposition.

Proposition 7.1. Denote $\Lambda^{n}=\left\|U^{n}\right\|_{L^{\infty}(\Omega)}$. Then under the conditions that $W^{n-1} \geq 0$ a.e. in $\Omega$ and $\tau_{n} \Lambda^{n}<$ 1, Equation (7.3) has a unique solution $W^{n} \in Q_{h}$ satisfying $W^{n} \geq 0$ a.e. in $\Omega$.

Proof. We observe first that (7.3) can be rewritten in the vector form as

$$
A \vec{W}^{n}=\vec{W}^{n-1}
$$

where $A$ is a matrix with positive diagonal and non-positive off-diagonal entries. For any $K \in \Sigma_{h}$ such that $\partial K_{-}^{n} \cap \Gamma=\emptyset$, the corresponding diagonal entry of $A$ is

$$
a_{K K}=1+\frac{\tau_{n}}{|K|} \int_{\partial K_{+}^{n}} \mathbf{P}^{n} \cdot \mathbf{n} \mathrm{d} \gamma
$$

and the sum of the off-diagonal entries is

$$
\sum_{L \neq K, L \in \Sigma_{h}} a_{L K}=\frac{\tau_{n}}{|K|} \int_{\partial K_{-}^{n}} \mathbf{P}^{n} \cdot \mathbf{n} \mathrm{d} \gamma
$$

Since $a_{L K} \leq 0$ for any $L \in \Sigma_{h}, L \neq K$, we have

$$
\begin{aligned}
\sum_{L \neq K, L \in \Sigma_{h}}\left|a_{L K}\right| & =-\sum_{L \neq K, L \in \Sigma_{h}} a_{L K} \\
& =-\frac{\tau_{n}}{|K|} \int_{\partial K_{-}^{n}} \mathbf{P}^{n} \cdot \mathbf{n} \mathrm{d} \gamma \\
& =-\frac{\tau_{n}}{|K|} \int_{\partial K^{n}} \mathbf{P}^{n} \cdot \mathbf{n} \mathrm{d} \gamma+\frac{\tau_{n}}{|K|} \int_{\partial K_{+}^{n}} \mathbf{P}^{n} \cdot \mathbf{n} \mathrm{d} \gamma \\
& =-\frac{\tau_{n}}{|K|} \int_{K} \operatorname{div} \mathbf{P}^{n} \mathrm{~d} \mathbf{x}+\frac{\tau_{n}}{|K|} \int_{\partial K_{+}^{n}} \mathbf{P}^{n} \cdot \mathbf{n} \mathrm{d} \gamma \\
& =\tau_{n}\left(U^{n}-W^{n-1}\right)+\frac{\tau_{n}}{|K|} \int_{\partial K_{+}^{n}} \mathbf{P}^{n} \cdot \mathbf{n} \mathrm{d} \gamma \\
& \leq \tau_{n} \Lambda^{n}+\frac{\tau_{n}}{|K|} \int_{\partial K_{+}^{n}} \mathbf{P}^{n} \cdot \mathbf{n} \mathrm{d} \gamma \\
& <a_{K K},
\end{aligned}
$$

where we have used (7.1) and the assumptions $W^{n-1} \geq 0$ a.e. in $\Omega$ and $\tau_{n} \Lambda^{n}<1$.

If $\partial K_{-}^{n} \cap \Gamma \neq \emptyset$, we still have (7.5) since now the sum of the absolute values of the off-diagonal entries is no greater than

$$
-\int_{\partial K_{-}^{n}} \mathbf{P}^{n} \cdot \mathbf{n} \mathrm{d} \gamma
$$

due to the definition $W^{n,-}=0$ on $\partial K_{-}^{n} \cap \Gamma$.

This proves that $A$ is an M-matrix. By the well-known property of M-matrices, we know that (7.3) has a unique solution $W^{n} \in Q_{h}$. Since $W^{n-1} \geq 0$, we deduce from (7.4) that $W^{n} \geq 0$. 
Now we can use the method in 5 to derive uniform stability estimates for the full-discrete solutions $\left(W^{n}, U^{n}, \mathbf{P}^{n}\right)$ and thus prove the convergence of the scheme (7.1-7.3). Here we omit the details.

Finally, we remark that in practical computations the condition $\tau_{n} \Lambda^{n}<1$ is not very restrictive since we have $\left\|W^{n}\right\|_{L^{\infty}(\Omega)} \leq c$ for some constant $c$ independent of $h$ and the time steps $\tau_{k}, k \leq n-1$.

\section{NUMERICAL EXPERIMENTS}

To test the mixed finite element approximation scheme presented in this paper, we implemented the algorithm (7.1-7.3) on a square domain $\Omega=[0, L]^{2}$. A simple triangulation is obtained by dividing each cell of a uniform cartisian grid of grid size $h$ along the $x=y$ diagonal. The step size is taken to be $\min \left\{\sigma h, 1 /\left(2\|u\|_{\infty}\right)\right\}$ for some properly chosen constant $\sigma$ (say $\sigma=0.5$ or $\sigma=2$ ). As our main purpose is to demonstrate that the numerical schemes presented in this paper indeed work, we only present a few simple cases. Results of more extensive simulations and their physical relevance with the motion of the superconducting vortices as well as numerical studies of the convergence rate of the numerical algorithms are to be reported elsewhere.

For any pair of two neighboring triangles that occupy the same square in the cartisian grid, we calculate the averaged values of the vortex densities $\omega_{h}$ and the averaged values of the magnetic field $u_{h}$ in the two triangles. Then, we use MATLAB to draw the surface plots of these averaged quantities.
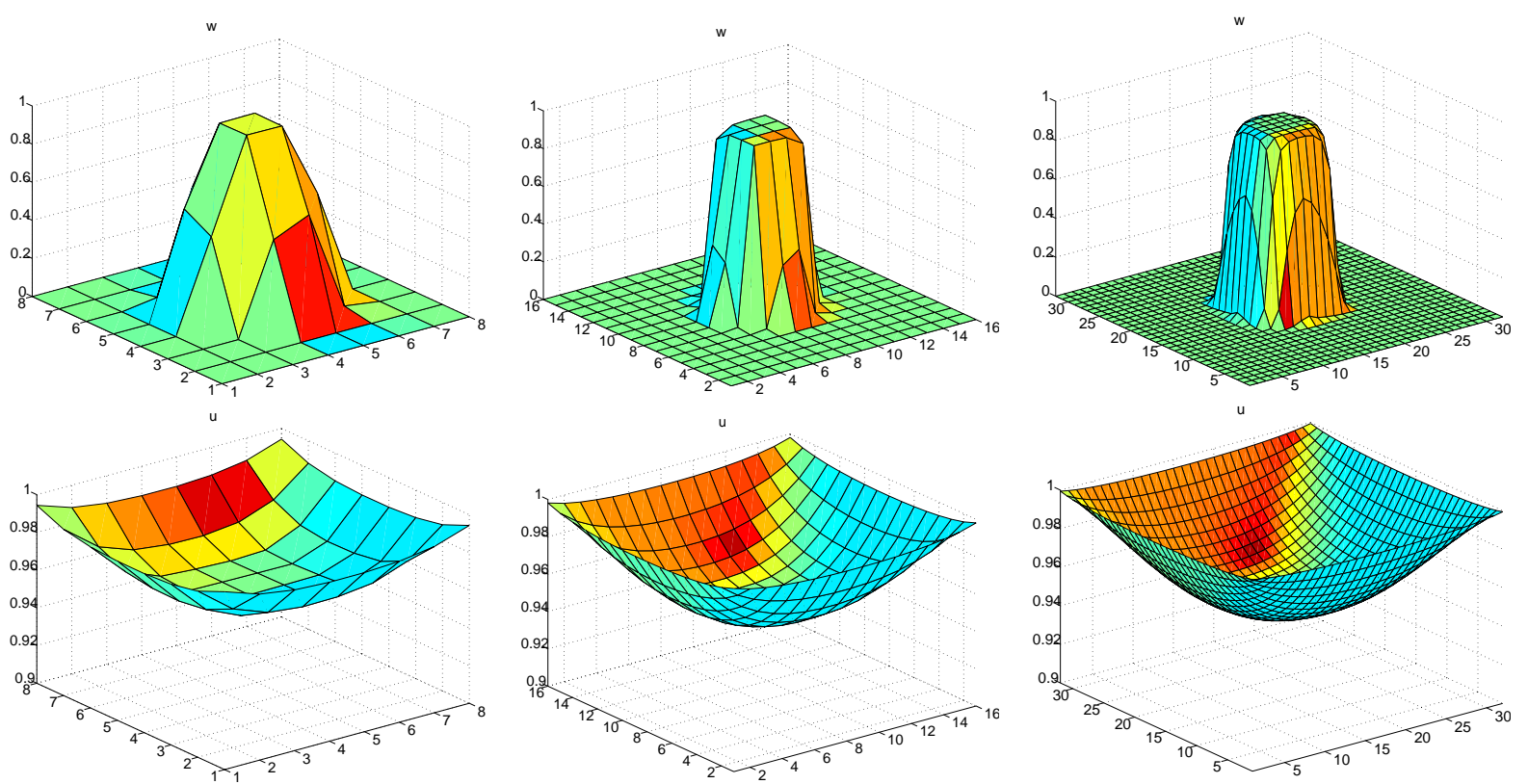

Figure 1. The steady state vortex densities (top) and the magnetic fields (bottom): $h=1 / 8,1 / 16,1 / 32$.

Case 1 . We first let $L=1$, we take the initial vortex density as

$$
\omega_{0}(x, y)=\left\{\begin{array}{ll}
1 & (x, y) \in\left\{\|(x, y)-(L / 2, L / 2)\|_{\infty} \leq L / 6\right\} \\
0 & \text { elsewhere }
\end{array} .\right.
$$

The applied field $H_{\text {ext }}=1$ is taken to be a constant field on the boundary.

As $t$ gets larger, the vortex density is approaching to a steady state. In Figure 1, the steady state vortex densities and the magnetic fields are shown for mesh with grid sizes $h=1 / 8, h=1 / 16$ and $h=1 / 32$. The convergence of the numerical solutions as $h \rightarrow 0$ is evident from the pictures. 

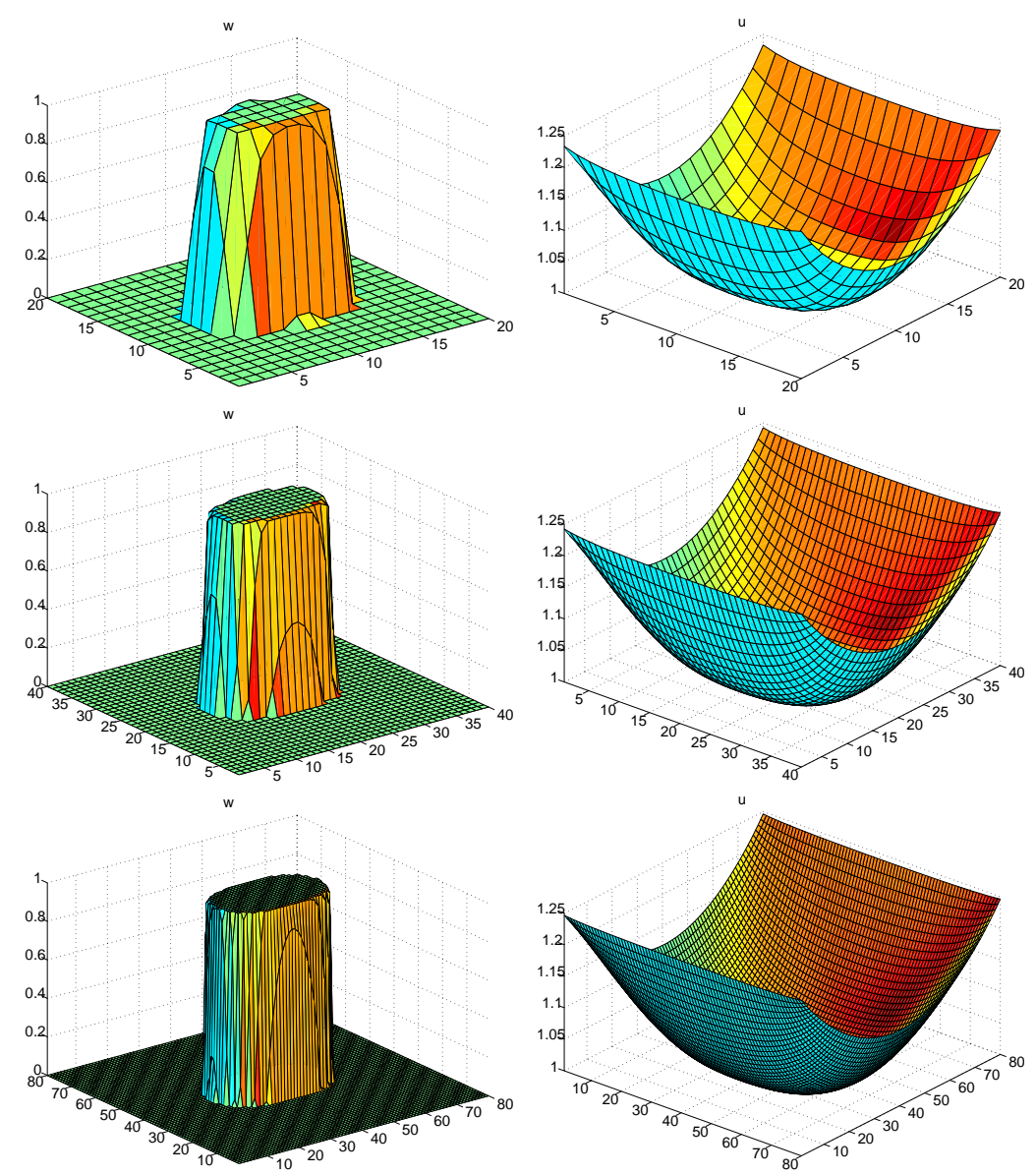

Figure 2. The steady state vortex densities (left) and the magnetic fields (right): $h=1 / 10,1 / 20,1 / 40$.

Case 2. Next, we let $L=2$, and take the initial vortex density as

$$
\omega_{0}(x, y)=\left\{\begin{array}{ll}
0.8 & (x, y) \in\left\{\|(x, y)-(L / 2, L / 2)\|_{\infty} \leq L / 6\right\} \\
0 & \text { elsewhere }
\end{array} .\right.
$$

We take a non-uniform applied field

$$
H_{\text {ext }}(x, y)=1.25-0.5 x(L-x) / L^{2}
$$

for any point $(x, y) \in \partial \Omega$.

As $t$ gets larger, the vortex density is approaching to a steady state. In the Figure 2, we present the steady states computed on grids with $h=1 / 10, h=1 / 20$ and $h=1 / 40$ respectively. Note that the region with nonzero vorticity becomes more elliptical due to the annisotropic applied field.

Case 3. Finally, we let $L=1$, and take the initial vortex density as

$$
\omega_{0}(x, y)=1, \quad \forall(x, y) \in \Omega .
$$


The applied field is taken to be a non-uniform field given on the boundary by:

$$
H_{\text {ext }}(x, y)=3-10(x(L-x)+y(L-y)) / L^{2}
$$

for any point $(x, y) \in \partial \Omega$. Note that the initial condition does not have a compact support. However, with the assumption that the vortex density is zero at all the in-flow boundary, the implementation of the algorithm is the same as in the compactly supported case.

The steady state vortex densities calculated on grids with $h=1 / 10, h=1 / 20$ and $h=1 / 40$ respectively are given in Figure 3.

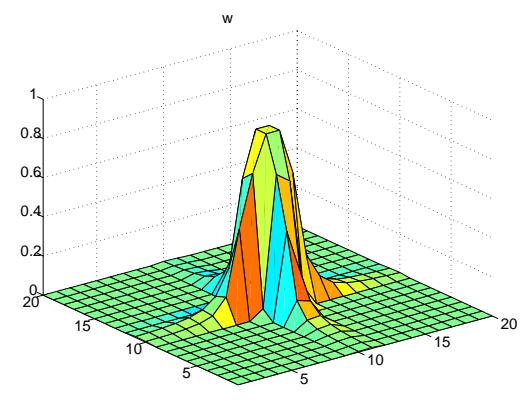

w
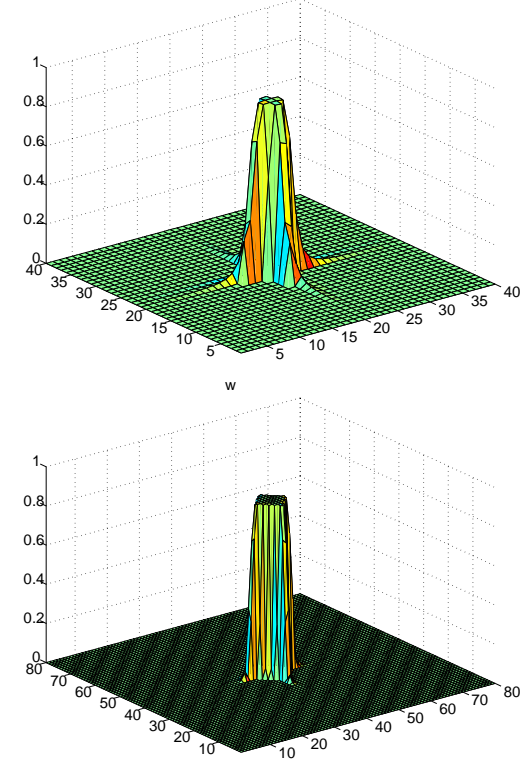
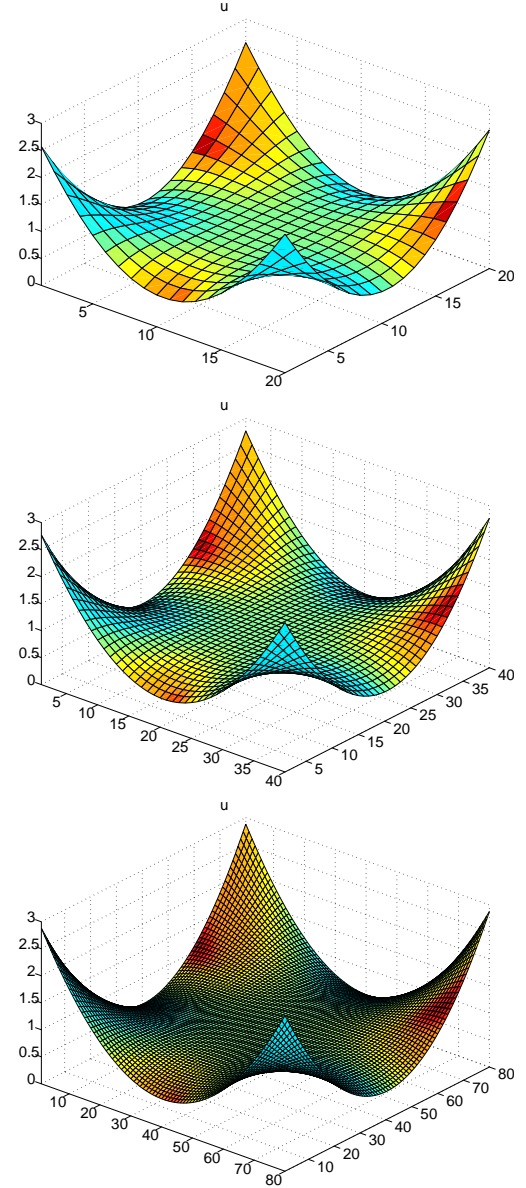

FiguRE 3. The steady state vortex densities (left) and the magnetic fields (right): $h=1 / 10,1 / 20,1 / 40$.

As implied by the zero in-flow boundary condition, while no vorticity is allowed to be nucleated at the boundary, vorticity may exit the domain through the out-flow boundary.

To indicate how the total vorticity

$$
V\left(t^{n}\right)=\int_{\Omega} W^{n} \mathrm{~d} \mathbf{x}
$$

decreases as time increases due to the exit of vorticity, we present a plot of values of $-\log \left(V\left(t^{n}\right)\right)$ with respect to the time changes in Figure 4.

The out-flow boundary, as determined by values of $\mathbf{P}^{n} \cdot \mathbf{n}$ on the boundary, are centered near the mid-points of the four sides of the square which can be observed from the plots given in the Figure 5 . 


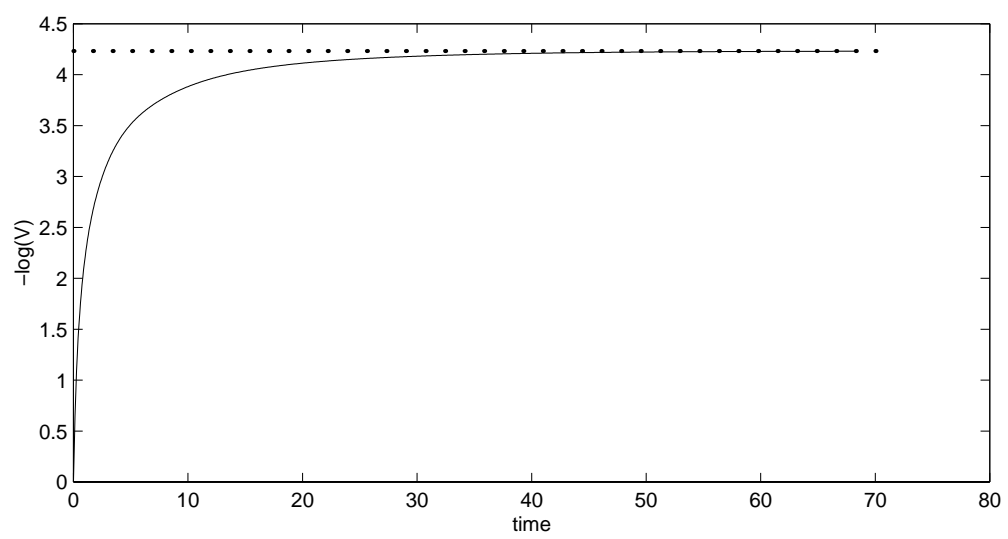

Figure 4. The plots of $-\log (W)$ in time (solid line) vesus the its steady state value (dotted line).
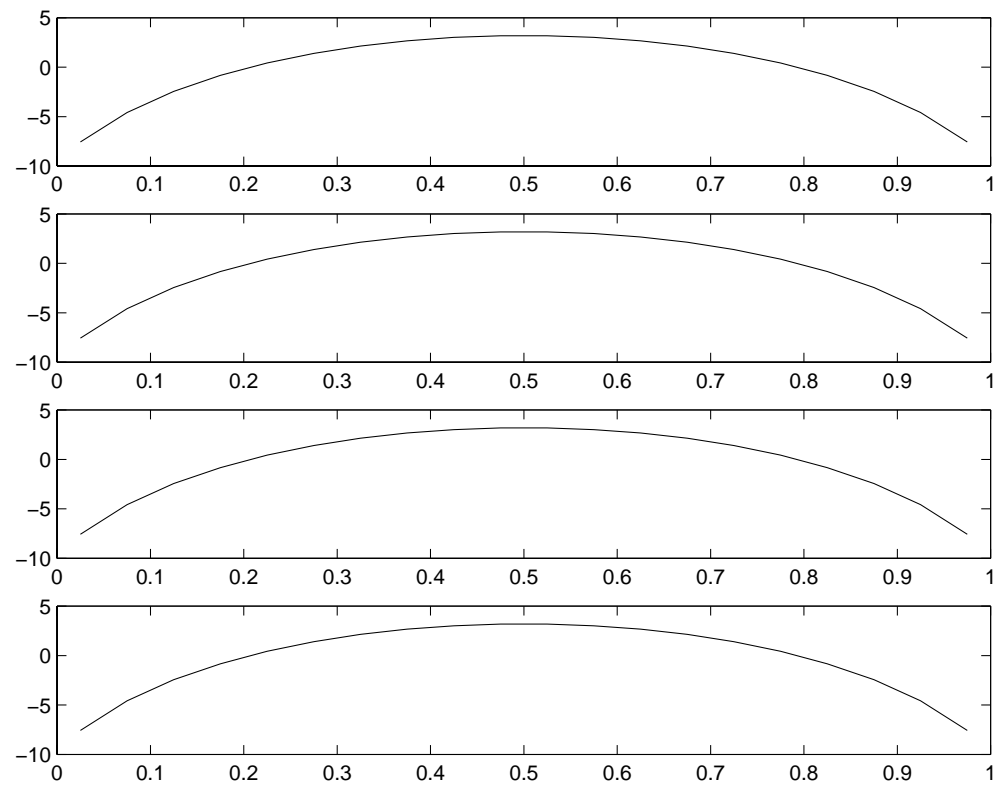

Figure 5. The steady state values of the normal derivatives $\mathbf{P}^{n} \cdot \mathbf{n}$ on the four sides of the square.

Case 4. Finally, we conduct a simulation for the annihilation of signed vortices. Although the model we have studied in this paper is only for the case of nonnegative vorticity, its generalization has been given in [3] to allow vorticity with sign changes. In this case the velocity field is given by $-\nabla u \operatorname{sgn}(\omega)$ where $\operatorname{sgn}$ is the standard sign function. We have implemented the non-conforming finite element scheme with this extension. At the time step $t_{n}$, the extra term $\operatorname{sgn}(\omega)$ is taken to be $\operatorname{sgn}\left(W^{n-1}\right)$. This leaves a linear system for $W^{n}$ once $U^{n}$ is computed.

In the experiment, we let $L=10$, and take the initial vortex density as

$$
\omega_{0}(x, y)=\sin (2 \pi x / L) \sin (2 \pi y / L), \quad \forall(x, y) \in \Omega
$$

The applied field is taken to be zero on all parts of the boundary. 
In Figure 6, we present the time evolution of the vorticity $\omega$ and the magnetic field $u$ computed using a grid size $h=L / 40$.
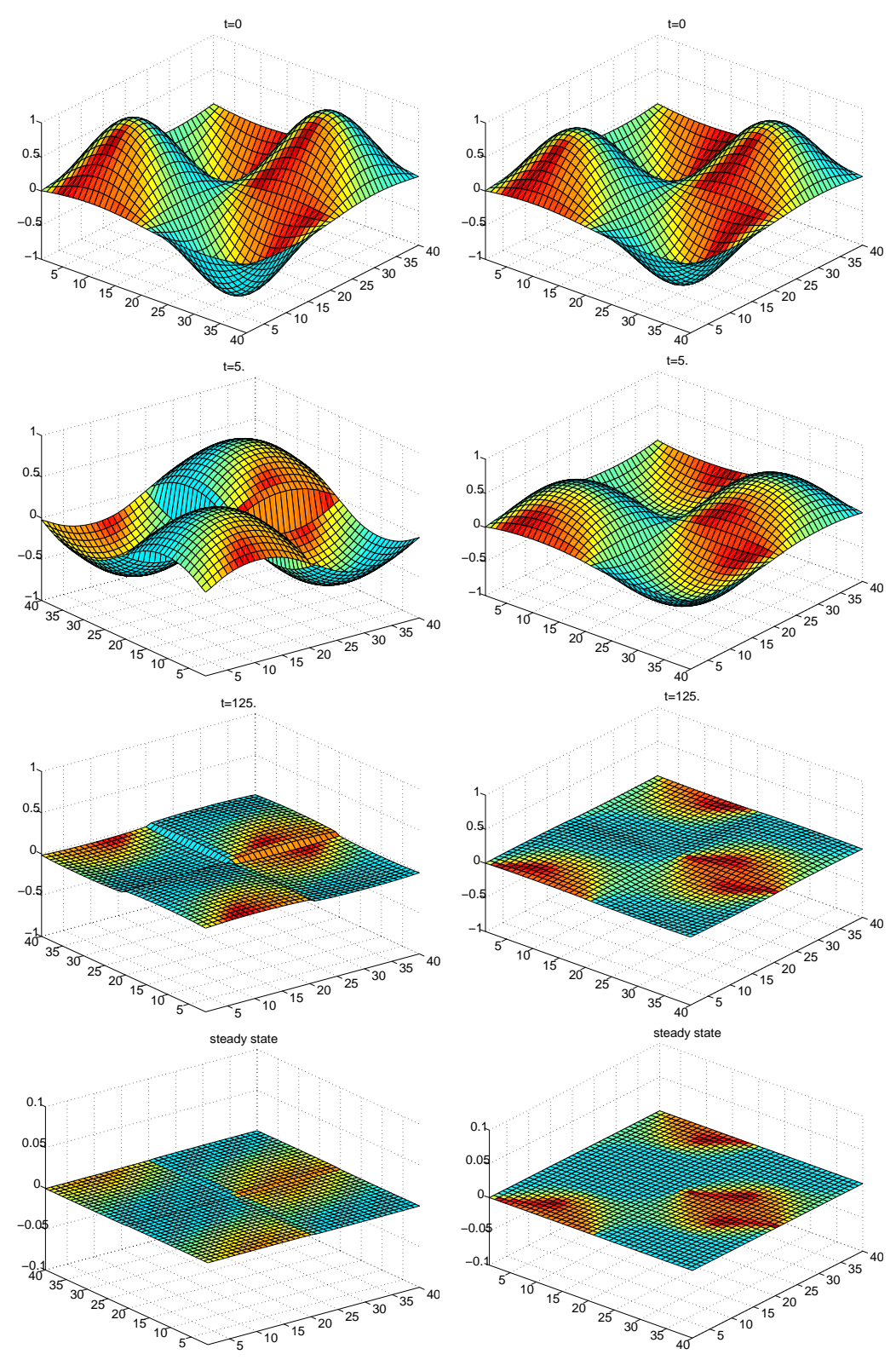

Figure 6. The time evolution of the vorticity $\omega$ (left) and the magnetic field $u$ (right).

As $t$ increases, we calculate the total vorticity

$$
V\left(t^{n}\right)=\int_{\Omega} W^{n} \mathrm{~d} \mathbf{x}
$$


and find that it remains as zero for all times. However, the magnitude of the vorticity

$$
M\left(t^{n}\right)=\left\|W^{n}\right\|_{\infty}
$$

starts to decrease due to the annihilation phenomena (see the simulation based on the Ginzburg-Landau models given in [12]). These results are shown in Figure 7 The solution eventually reaches the steady state for which both the vorticity and the magnetic field are zeros uniformly.
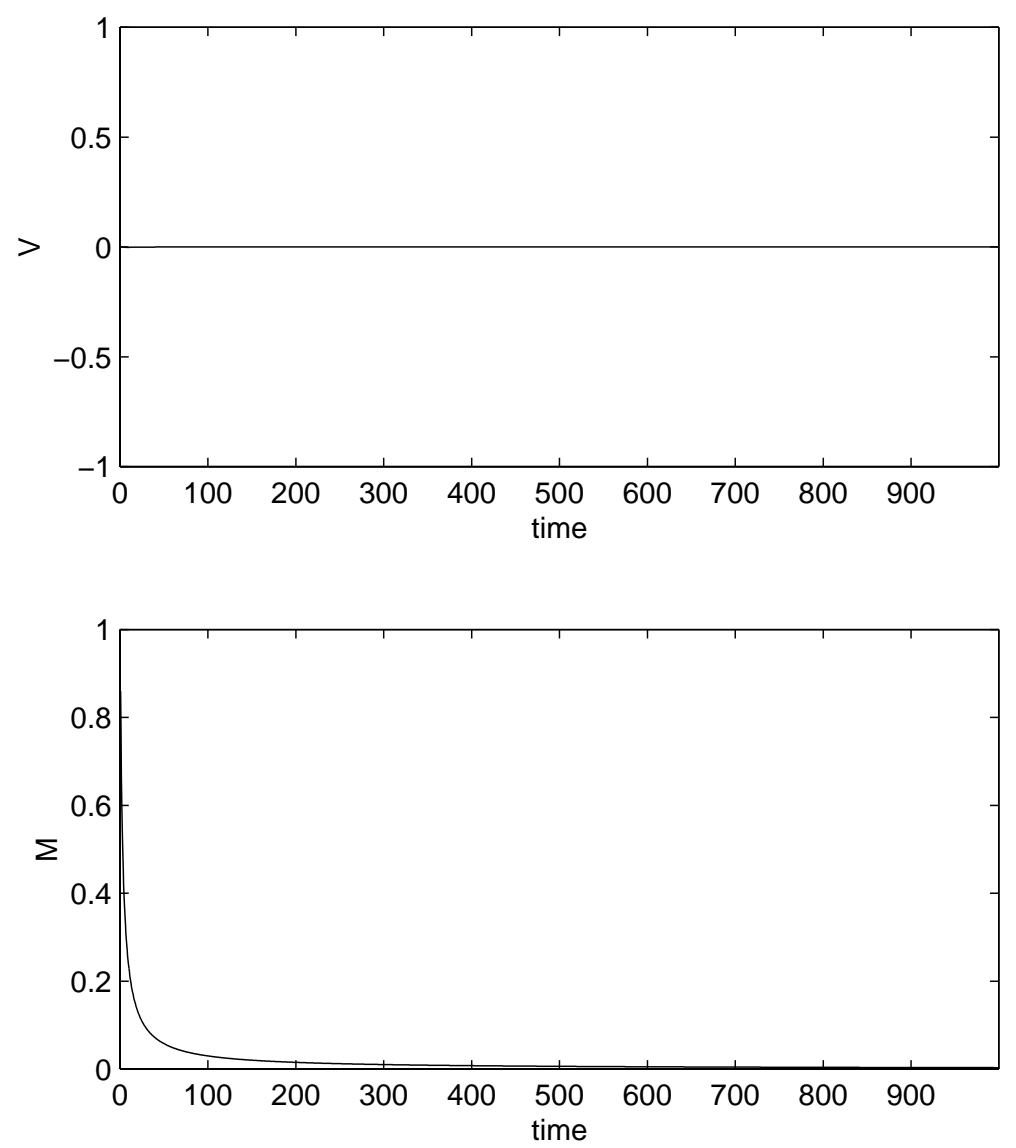

Figure 7. The conservation of $V(t)$ and the decay of the $M(t)$ in time.

We have repeated the above experiment on grids with grid size $h=L / 80$ and $h=L / 160$ and the convergence of the numerical solutions have been observed although our convergence theory does not apply to this case.

Remark 8.1. (Remark on the numerical experiments) The above numerical experiments indicate that the implementation of the numerical schemes presented in this paper is very successful. One may also observe the interesting phenomena concerning the finite time exit of vorticity on the boundary and the annihilation of signed vorticity. More simulations on the solutions of the mean field model for superconducting vortices and their comparisons with the conventional Ginzburg-Landau simulations will be carried out in the future.

\section{Conclusion}

In this paper, a convergence analysis is given for both semi-discrete and fully-discrete upwinding mixed finite element approximations of a mean field model in a simple situation. The theory can be successfully applied 
to cases where an unstructured triangular grid is required to solve the model in irregular domains (domains other than rectangles). Results of some preliminary numerical tests seem very encouraging. We also remark that the upwinding scheme proposed in this paper to solve the vortex density equation (1.1) is of first order since the vortex density is approximated by piecewise constant element. However, by combining with the highorder Runge-Kutta local projection discontinuous Galerkin method proposed for conservation laws on general triangulation [7], one might construct high order schemes for (1.1) by solving the elliptic equation (1.2) with high order mixed finite elements such as Raviart-Thomas element $\mathrm{RT}_{k}$ or Brezzi-Douglas-Marini element $\mathrm{BDM}_{k}$ which have been extensively studied in [2].

An advantage of using the mean field model in simulating vortex dynamics in superconductors, compared to using the Ginzburg-Landau models, is the ability to consider large scale vortex interactions without resolving the fine structures of individual vortices. Here, we have also only considered a simplified model for which the pinning effect and the vortex nucleation are neglected. In the more general case, the velocity of the vorticity transport also depends on the pinning forces and the critical current. The mathematical understanding of the more general models is very limited so far, moreover, there are still gaps in a rigorous justification of the formal derivations of the mean field model from the asymptotic limits of the Ginzburg-Landau equations. Thus, we expect to conduct more numerical simulations that would provide some comparison with the Ginzburg-Landau simulations as well as possible numerical justification of the mean field models in the future.

\section{REFERENCES}

[1] S.C. Brenner and L.R. Scott, The mathematical theory of finite element methods. Springer-Verlag, New York (1994).

[2] F. Brezzi and M. Fortin, Mixed and Hybrid Finite Element Methods. Springer, New York (1991).

[3] S.J. Chapman, A mean-field model of superconducting vortices in three dimensions. SIAM J. Appl. Math. 55 (1995) $1259-1274$.

[4] S.J. Chapman and G. Richardson, Motion of vortices in type-II superconductors. SIAM J. Appl. Math. 55 (1995) $1275-1296$.

[5] S.J. Chapman, J. Rubenstein, and M. Schatzman, A mean-field model of superconducting vortices. Euro. J. Appl. Math. 7 (1996) 97-111.

[6] Z. Chen and S. Dai, Adaptive Galerkin methods with error control for a dynamical Ginzburg-Landau model in superconductivity. (Preprint, 1998).

[7] B. Cockburn, S. Hou and C.-W. Shu, The Runge-Kutta local projection discontinuos galerkin finite element method for conservation laws IV: The multidimensional case. Math. Com. 54 (1990) 545-581.

[8] Q. Du, Convergence analysis of a hybrid numerical method for a mean field model of superconducting vortices. SIAM Numer. Analysis, (1998).

[9] Q. Du, M. Gunzburger, and J. Peterson, Analysis and approximation of the Ginzburg-Landau model of superconductivity. SIAM Review 34 (1992) 54-81.

[10] Q. Du, M. Gunzburger, and J. Peterson, Computational simulations of type-II superconductivity including pinning mechanisms. Phys. Rev. B 51 (1995) 16194-16203.

[11] Q. Du, M. Gunzburger and H. Lee, Analysis and computation of a mean field model for superconductivity. Numer. Math. 81 539-560 (1999).

[12] Q. Du and Gray, High-kappa limit of the time dependent Ginzburg-Landau model for superconductivity. SIAM J. Appl. Math. 56 (1996) 1060-1093.

[13] W. E, Dynamics of vortices in Ginzburg-Landau theories with applications to superconductivity. Phys. D 77 (1994) $383-404$.

[14] C. Elliott and V. Styles, Numerical analysis of a mean field model of superconductivity. preprint.

[15] V. Girault and -A. Raviart, Finite Element Methods for Navier-Stokes Equations. Springer, Berlin (1986).

[16] Grisvard, Elliptic Problems on Non-smooth Domains. Pitman, Boston (1985).

[17] C. Huang and T. Svobodny, Evolution of Mixed-state Regions in type-II superconductors. SIAM J. Math. Anal. 29 (1998) 1002-1021.

[18] Lesaint and P.A. Raviart, On a Finite Element Method for Solving the Neutron Transport equation, in: Mathematical Aspects of the Finite Element Method in Partial Differential Equations, C. de Boor Ed., Academic Press, New York (1974).

[19] L. Prigozhin, On the Bean critical-state model of superconductivity. Euro. J. Appl. Math. 7 (1996) $237-247$.

[20] L. Prigozhin, The Bean model in superconductivity: variational formulation and numerical solution. J. Com Phys. 129 (1996) 190-200.

[21] Raviart and J. Thomas, A mixed element method for 2nd order elliptic problems, in: Mathematical Aspects of the Finite Element Method, Lecture Notes on Mathematics, Springer, Berlin 606 (1977).

[22] R. Schatale and V. Styles, Analysis of a mean field model of superconducting vortices (preprint).

[23] R. Temam, Navier-Stokes equations, Theory and Numerical Analysis. North-Holland, Amsterdam (1984). 